\title{
Relativistic Dirac-Fock-Slater program to calculate potential-energy curves for diatomic molecules
}

\author{
W. -D. Sepp, D. Kolb, W. Sengler, H. Hartung, and B. Fricke \\ Department of Physics, University of Kassel, D-3500 Kassel, West Germany \\ (Received 8 August 1985)
}

\begin{abstract}
A LCAO-MO (linear combination of atomic orbitals-molecular orbitals) relativistic Dirac-FockSlater program is presented, which allows one to calculate accurate total energies for diatomic molecules. Numerical atomic Dirac-Fock-Slater wave functions are used as basis functions. All integrations as well as the solution of the Poisson equation are done fully numerical, with a relative accuracy of $10^{-5}-10^{-6}$. The details of the method as well as first results are presented here.
\end{abstract}

\section{INTRODUCTION}

The fast development of computers during the last 30 years made it possible to calculate more and more complex atomic and molecular systems with increasingly better methods and accuracy. All types of atomic Hartree-Fock, ${ }^{1,2}$ and random-phase approximation (RPA) programs ${ }^{3}$ have been developed as nonrelativistic codes, whereas analogue Dirac-Fock ${ }^{4-6}$ (DF) and relativistic random-phase approximation (RRPA) programs ${ }^{7}$ are in use as relativistic codes. All these atomic codes [with the exception of that by Kim, Ref. 5(c)] use the direct integration of the Schrödinger or Dirac equation with finite difference methods.

The current available quantum-chemical codes for molecules use basis-set expansion methods with (in principle) Slater- or Gauss-type basis functions. A large variety of such quantum-chemical codes are established and lead to very accurate results, but all these codes for molecules have in common that they are nonrelativistic. A nearly complete list of references, which covers the whole field of quantum-chemical calculations, is given in the book by Schaefer. ${ }^{8}$ This book also includes the principal references for pseudopotential calculations, ${ }^{9}$ as well as the beginning of seminumerical, two-dimensional calculations by $\mathrm{McCullough}^{10}$ and their extension to diatomic multiconfiguration self-consistent-field (MCSCF) wave functions. ${ }^{11}$ More recent extensions to fully numerical twodimensional Hartree-Fock-Slater (HFS) calculations are given by Becke ${ }^{12}$ as well as Laaksonen et al. ${ }^{13}$

Several groups persevere in trying to establish analogue relativistic quantum-chemical calculations. Reference 14 summarizes this whole field up to 1982 . The paper by Kutzelnigg ${ }^{15}$ reviews the problems which arise in the relativistic formulation. Actual relativistic DF calculations are presented in Ref. 16. First attempts to solve the relativistic two-dimensional problem numerically are given in Ref. 17 and 18. Up until now, it has been practically impossible to calculate small molecules containing very heavy atoms on a Dirac-Fock level, with the exception of heavy hydrides such as $\mathrm{PbH}_{4}$, which were calculated with a one-center expansion method. ${ }^{19}$ For this reason approximative methods such as the relativistic pseudopotential approaches,${ }^{20}$ a perturbation treatment of relativistic effects on top of HFS calculations, ${ }^{21}$ relativistic scatteredwave calculations, ${ }^{22}$ or relativistic Dirac-Fock-Slater (DFS) calculations, are essential to provide useful information in a region of elements where more accurate calculations are not feasible.

We present here a relativistic DFS program for diatomic molecules, which uses numerical atomic DFS wave functions as basis functions. The development of this program originates from the work of Rosén and Ellis ${ }^{23}$ who first developed a relativistic self-consistent-charge (SCC) code, which itself followed the ideas of the nonrelativistic SCC method with numerical basis functions. ${ }^{24}$ The drawback with these calculations was the inherent noise existing in the discrete variational method, ${ }^{25}$ which was used to calculate the matrix elements. Because of the relative accuracy of these calculations in the order of percent, it was possible to discuss only level schemes for molecules at chemical distances ${ }^{26}$ and one-electron correlation diagrams in heavy-ion scattering for distances down to the united atom limit. ${ }^{27}$

The new approach is threefold: First, we solve the two-dimensional Poisson equation numerically with a relative accuracy of $10^{-5}-10^{-6}$. (An alternative approach would be a multicenter expansion as used by Delley and Ellis $^{28}$ in nonrelativistic calculations.) Second, the numerically calculated matrix elements are improved in accuracy by 3 to 4 orders of magnitude. Third, the next improvement is the possibility of including atomic wave functions as basis functions, which are generated in the monopole part of the molecular potential. This last improvement is essential for the calculation of quasimolecules at small internuclear distances. In addition, various further basis sets at various sites can be introduced.

This program is thus accurate enough to obtain physically useful, self-consistent-field (SCF) results for the potential-energy curve, energy eigenvalues, and wave functions for diatomic molecules and quasimolecules at internuclear distances between zero and chemical distances.

This paper is organized as follows. In Sec. II the relativistic DFS method is discussed, and in Sec. III the nu- 
merical Gauss-Laguerre integration method as well as the two-dimensional solution of the Poisson equation are discussed. In Secs. IV and V the choice of our basis functions, respectively, the preorthogonalization is presented. Section VI contains the first results of the DFS calculations for the case of $\mathrm{N}_{2}$ as a chemically bound system, and $\mathrm{Ne}-\mathrm{Ne}$ as a case where elastic scattering data are available for comparison. The advantages and disadvantages of the method as well as future developments will be presented in Sec. VIII.

\section{THE RELATIVISTIC DFS METHOD}

The general method of a relativistic DFS calculation in molecules has been described in various papers. ${ }^{23,24,28,29}$ Therefore, we only wish to summarize the method briefly. The relativistic many-electron configuration-space DiracHamiltonian of a molecular system is usually written as

$$
\hat{H}_{C S}=\sum_{j=1}^{N} \hat{h}_{j}+\sum_{\substack{i, j=1 \\ i>j}}^{N} \hat{V}_{i j},
$$

where $\hat{h}_{i}$ is the single-particle Hamiltonian

$$
\hat{h}_{j}=c \alpha_{j} \mathbf{p}_{j}+\beta_{j} m c^{2}+\hat{V}_{j}^{\text {nuc }}
$$

with the Dirac matrices

$$
\alpha_{j}=\left(\begin{array}{cc}
0 & \sigma_{j} \\
\sigma_{j} & 0
\end{array}\right)
$$

and

$$
\beta_{j}=\left(\begin{array}{cc}
I_{j} & 0 \\
0 & -I_{j}
\end{array}\right)
$$

where $\sigma$ and $I$ are the three standard $2 \times 2$ Pauli matrices, and the $2 \times 2$ unit matrix, respectively. The operator $\hat{V}_{j}^{\text {nuc }}$ represents the electron-nucleus interaction energy

$$
\hat{V}_{j}^{\text {nuc }}=-e^{2} \sum_{K} \frac{Z_{K}}{\left|\mathbf{r}_{j}-\mathbf{R}_{K}\right|},
$$

and $\hat{V}_{i j}$ is the electron-electron Coulomb interaction energy

$$
\hat{V}_{i j}=\frac{e^{2}}{\left|\mathbf{r}_{i}-\mathbf{r}_{j}\right|} .
$$

$N$ is the number of the electrons. Not included in the Hamiltonian (1) is, of course, the Breit interaction, because uncertainties of the Slater approximation are already larger than the effect of the Breit term would be and it would also complicate the whole calculation much more. Not included as well are projection operators first proposed by Brown and Ravenhall. ${ }^{30}$ Although it is not yet understood in detail, we think that spurious positron contributions should be small in our calculations.

The DFS method uses two approximations: At first the wave function is taken to be a single Slater determinant, and then the electron-exchange term is approximated by the $X_{\alpha}$ method (in all our calculations we use $X_{\alpha}=0.7$ ).

The DFS equation then reads

$$
\hat{h}^{\mathrm{DFS}} \psi_{v}(\mathrm{r}, s)=\varepsilon_{v} \psi_{v}(\mathrm{r}, s)
$$

with $\hat{h}^{\mathrm{DFS}}$ the effective one-particle DFS operator

$$
\hat{h}^{\mathrm{DFS}}=\hat{t}+\hat{V}^{\mathrm{nuc}}+\hat{V}^{C}+\hat{V}^{\mathrm{ex}},
$$

where the kinetic operator $\hat{t}$, and the electron-nucleus operator $\widehat{V}^{\text {nuc }}$ have the same form as above [Eqs. (2) and (3)].

The direct potential part is given by

$$
\hat{V}^{c}(\mathbf{r})=\int \mathbf{d} \mathbf{r}^{\prime} \frac{\rho\left(\mathbf{r}^{\prime}\right)}{\left|\mathbf{r}-\mathbf{r}^{\prime}\right|},
$$

and the exchange potential part by

$$
\hat{V}^{\mathrm{ex}}(\mathbf{r})=-3 X_{\alpha}\left[\frac{3}{8 \pi} \rho(\mathrm{r})\right]^{1 / 3}
$$

with the electron density

$$
\rho(\mathbf{r})=e^{2} \sum_{\nu \in o c c} \psi_{v}^{+}(\mathbf{r}, s) \psi_{v}(\mathbf{r}, s) .
$$

The solution of the $N$-coupled differential equations (5) has to be found by SCF iteration. To actually solve these equations for the two-dimensional case several methods are possible. The first is the direct numerical integration of the differential equation. This method is not available in updated form except for very first trials ${ }^{17,18}$ for the system $\mathrm{H}_{2}{ }^{+}$.

The second method is to use a multipole expansion of the wave functions in the angular variables; this leads to an even larger set of coupled equations, that are however, one dimensional. This method was used first in the relativistic case for a pure two-center point-Coulomb potential by Betz. ${ }^{31}$

The third method is the expansion of the molecular wave functions into basis functions, usually called MOLCAO (molecular orbitals-linear combination of atomic orbitals). The wave functions $\psi_{v}$ are expanded in symmetry orbitals $\chi_{\lambda}$

$$
\psi_{\nu}(\mathbf{r})=\sum_{\lambda} c_{\nu \lambda} \chi_{\lambda}
$$

and the symmetry orbitals are expanded into atomic basis functions $\varphi_{k}$

$$
\chi_{\lambda}=\sum_{k} d_{\lambda k} \varphi_{k}
$$

If we insert this in Eq. (5), the DFS equations reduce to the matrix eigenvalue problem

$$
\underline{h c}^{T}=\underline{\varepsilon S c}^{T}
$$

with the Fock matrix

$$
\underline{h}=\left(h_{\lambda \mu}\right) \text { with } h_{\lambda \mu}=\left\langle\chi_{\lambda}|\hat{h}| \chi_{\mu}\right\rangle,
$$

the overlap matrix

$$
\underline{S}=\left(S_{\lambda_{\mu}}\right) \text { with } S_{\lambda_{\mu}}=\left\langle\chi_{\lambda} \mid \chi_{\mu}\right\rangle,
$$

the coefficient matrix

$$
\underline{c} \text { with } c_{\nu \lambda}=\left(c^{T}\right)_{\lambda_{\nu}},
$$

and the eigenvalue matrix 


$$
\underline{\varepsilon}=\left(\varepsilon_{v \lambda}\right) \text { and } \varepsilon_{v \lambda}=\delta_{v \lambda} \varepsilon_{v} .
$$

The charge density can then be written

$$
\rho(r)=e^{2} \sum_{\lambda, \mu} \chi_{\lambda}^{+} \chi_{\mu} \sum_{i<F} c_{i \lambda}{ }^{\dagger} q_{i} c_{i \mu}=e^{2} \sum_{\lambda, \mu} \rho_{\lambda \mu} q_{\lambda \mu},
$$

with $\rho_{\lambda \mu}(r)$ the local-density matrix and $q_{\lambda \mu}$ the charge matrix. Again we can distinguish between two possibilities. The first possibility is the expansion of the atomic orbitals into some kind of Slater- or Gauss-type function, which is used with great success in nearly all nonrelativistic as well as relativistic molecular calculations. The advantage of this method is that all matrix elements used in the calculation can be calculated analytically with very high accuracy, so that the nonorthogonality of the basis does not cause any problems. The disadvantage is that the basis functions are relatively ill-adapted to the physical problem, so that a large number has to be used. The second possibility, which we are using here, is the choice of numerical atomic DFS wave functions as the basis. The disadvantage of this choice is that all matrix elements have to be calculated numerically, but the advantage is that the relatively small basis set is sufficient, and the contributions of the negative continuum will probably be small. In addition, the kinetic energy matrix elements can be calculated in this special numerical basis by a simple integration (thus avoiding numerical differentiation). ${ }^{23,26}$

\section{NUMERICAL INTEGRATION AND SOLUTION OF THE POISSON EQUATION}

The overlap- and Fock-matrix elements $\left\langle\chi_{i}|\hat{o}| \chi_{j}\right\rangle$ in Eq. (12) with $\hat{o}=I, \hat{h}$ are calculated numerically in realspace coordinates. As the integrands of the two-center problem are cylindrically symmetric, it is possible to separate the angular integration around the internuclear axis. The remaining two-dimensional integration is done in elliptic-hyperbolic coordinates

$$
\begin{aligned}
& \cosh \eta=\frac{r_{1}+r_{2}}{R}, 0 \leq \eta<\infty \\
& \cos \theta=\frac{r_{1}-r_{2}}{R}, 0 \leq \theta<\pi
\end{aligned}
$$

with $R$ the internuclear distance and $r_{i}$ the distance from the nuclei. The new "radial" coordinate $\cosh \eta$ is then focused by the transformation

$$
\cosh \eta=w\left(A-B e^{-C w}\right)+1,0 \leq w<\infty
$$

to achieve that for a given Gauss-Laguerre integration grid for $w$ the inner $\eta$ values lie densely enough to integrate the inner-shell contributions, and the outermost points are put into the region where the outer-shell wave functions decrease exponentially. The "radial" GaussLaguerre integration scheme over $w$ uses about 35 points, which is optimal for the integration of exponentially decreasing functions from zero to infinity. The "angular" integration over $\cos \theta$ is done with a Gauss-Legendre method with about 40 points. Within this grid we get the overlap- and Fock-matrix elements with a relative error of $10^{-5}$ to $10^{-6}$.

The direct part $V^{C}$ of the electron-electron potential
[Eq. (7)] is calculated from the electron density $\rho$ by numerical solution of Poisson's equation in elliptichyperbolic coordinates

$$
\hat{D} V^{C}=\rho
$$

with

$$
\hat{D}=-\frac{\frac{\partial^{2}}{\partial \eta^{2}}+\operatorname{coth} \eta \frac{\partial}{\partial \eta}+\frac{\partial^{2}}{\partial \theta^{2}}+\cot \theta \frac{\partial}{\partial \theta}}{4 \pi a^{2}\left(\sinh ^{2} \eta+\sin ^{2} \theta\right)}
$$

and $a=R / 2$. To avoid the singularities for $\eta \rightarrow 0$ and $\theta \rightarrow 0$, we use the variables $\cosh \eta$ and $\cos \theta$. For the discretization of $(20)$ we transformed the variables [analogue to Eq. (15)] to

$$
\begin{aligned}
& \cosh \eta=-E x e^{-F x}+1, \\
& \cos \theta=G\left(\frac{1}{2}-\frac{1}{1+e^{y / H}}\right) .
\end{aligned}
$$

The parameters $E, F, G, H$ are adjusted in such a way that the density $\rho(r)$ and the potential $V^{C}(r)$ are smoothly varying functions over the whole range of the $(x, y)$ plane. In this grid we solve the discreticized Poisson equation using a third-order finite difference method of Schwarztrauber and Sweet, ${ }^{32}$ which reduces the problem to solving a band-structured matrix equation.

A large improvement in accuracy is obtained by applying this method iteratively: The numerical solution of Poisson's equation for a given $\rho$ leads to the potential $V^{C}=\hat{D}^{-1} \rho$. Using a higher-order differentiation $D^{m}$, we calculate back $\rho^{\prime}=\hat{D}^{m} V^{C}$, which differs slightly from $\rho$ due to the more accurate numerical treatment. The difference density $\delta \rho=\rho-\rho^{\prime}$ gives a correction to the potential by numerical solution of the new Poisson equation $\delta V^{C}=\widehat{D}^{-1} \delta \rho$. With the new potential $V_{n}^{C}=V^{C}+\delta V^{C}$, this procedure can be repeated until $\delta V^{C}$ becomes insignificant. Empirically, with a sixth-order differentiation, the convergence point is reached already after two corrective iterations, and the resulting potential has an overall relative accuracy better than $10^{-5}-10^{-6}$ for an $(x, y)$ grid of $100 \times 100$ points for a diatomic system.

\section{CHOICE OF THE BASIS FUNCTIONS}

The choice of the basis functions used in the calculations is not straightforward. The first approximation to this problem is the choice of the minimal basis set, consisting of fully or partially occupied atomic levels of the separated atoms.

A number of optimized Slater and Gauss-orbitals, respectively, which are dependent on the system and the type of binding of the system, are usually added in the nonrelativistic quantum-chemical calculations. Optimized sets of basis functions are not yet available in the relativistic case. To learn more about the choice of basis functions we selected the simplest system $\mathrm{H}_{2}{ }^{+}$. Table I shows the convergence of the ground-state eigenvalues at the internuclear distance $R=1.0$ a.u. as function of the number of additional basis functions given on the left. In the last "row" we added a basis function called "mono- 
TABLE I. Energy eigenvalues in a.u. of the $\mathrm{H}_{2}{ }^{+}$ground state at $R=1$ a.u. as function of various sets of basis functions. The superscript mon means that these wave functions have been calculated in an atomic DFS calculation with the monopole part of the molecule as nuclear potential.

\begin{tabular}{|c|c|c|c|}
\hline Basis states & & & $\begin{array}{l}\text { Eigenvalue } \\
-\varepsilon_{\log } \text { (a.u.) }\end{array}$ \\
\hline $\begin{array}{l}\text { 2-center basis } \\
2\left(\mathrm{H}_{1 s}\right) \\
2\left(\mathrm{H}_{1 s, 2 s, 2 p}\right)\end{array}$ & 2 & 0 & $\begin{array}{l}1.28838 \\
1.31563\end{array}$ \\
\hline $\begin{array}{l}\text { 3-center basis } \\
2\left(\mathrm{H}_{1 s, 2 s, 2 p}\right)+\mathrm{He}_{3 s}^{\text {mon }} \\
2\left(\mathrm{H}_{1 s-3 d}\right)+\mathrm{He}_{3 s, 3 p, 3 d}^{\text {mon }}\end{array}$ & 2 & 1 & $\begin{array}{l}1.32181 \\
1.34921\end{array}$ \\
\hline $\begin{array}{l}\text { 4-center basis } \\
2\left(\mathrm{H}_{1 s}+\mathrm{He}_{1 s}\right) \\
2\left(\mathrm{H}_{1 s}+\mathrm{He}_{2 s}\right) \\
2\left(\mathrm{H}_{1 s}+\mathrm{He}_{1 s, 2 s}\right)\end{array}$ & 4 & 0 & $\begin{array}{l}1.42104 \\
1.42677 \\
1.43893\end{array}$ \\
\hline $\begin{array}{l}\text { 6-center basis } \\
2\left(\mathrm{H}_{1 s}+\mathrm{He}_{1 s, 2 s, 2 p}+\mathrm{Li}_{2 s, 2 p, 3 p}\right)\end{array}$ & 6 & 0 & 1.45045 \\
\hline $\begin{array}{l}\text { 11-center basis } \\
2\left(\mathrm{H}_{1 s}+\mathrm{He}_{1 s, 2 s, 2 p}+\mathrm{Li}_{2 s, 2 p, 3 p}\right. \\
\left.\quad+\mathrm{Be}_{3 p}+\mathrm{B}_{3 d}\right)+\mathrm{He}_{2 s, 2 p, 3 s, 3 p}^{\operatorname{mon}}\end{array}$ & 10 & 1 & 1.45113 \\
\hline $\begin{array}{l}\text { Exact value from the analytic } \\
\text { nonrelativistic solution }\end{array}$ & & & 1.45179 \\
\hline
\end{tabular}

pole function," as it is the solution of the DFS problem of an atom with the monopole part of the potential of the two nuclei. This last row also shows that the exact energy eigenvalue can be reproduced already within less than $0.5 \%$. Table II gives the energy eigenvalues and total energies of the same system $\mathrm{H}_{2}{ }^{+}$, with the basis from the last row of Table I as function of the internuclear distance. Table II shows an agreement always within the order of $10^{-4}$, although we certainly did not fully optimize the basis set. The choice of the basis functions for the calculations presented in Sec. VII was done in an analogue way. Of course, there is still a lot of work to be put into this question in the near future.

\section{PREORTHONORMALIZATION}

As one sees from the basis sets used here, the basis functions are not orthogonal. It is well known that such nonorthogonal basis sets usually produce a large error enhancement. As our numerical accuracy is only in the order of $10^{-6}$, we have to preothonormalize the basis before solving the eigenvalue problem. Orthonormalization

TABLE II. Energy eigenvalues and total energies of the $\mathrm{H}_{2}{ }^{+}$ground state for the 11 center basis for various internuclear distances.

\begin{tabular}{cccccc}
\hline \hline$R$ & $\begin{array}{c}-\varepsilon_{1 \sigma g} \\
\text { (a.u.) }\end{array}$ & $\begin{array}{c}-\varepsilon_{\text {log }} \text { (a.u.) } \\
\text { exact }\end{array}$ & $\Delta(\%)$ & $-E_{T}(\mathrm{eV})$ & $\begin{array}{c}E_{T}(\mathrm{eV}) \\
\text { exact }\end{array}$ \\
\hline 1.0 & 1.45113 & 1.45179 & $<0.46$ & 12.276 & 12.294 \\
1.5 & 1.24863 & 1.24899 & $<0.29$ & 15.836 & 15.846 \\
2.0 & 1.10235 & 1.10263 & $<0.26$ & 16.391 & 16.399 \\
2.5 & 0.99368 & 0.99382 & $<0.15$ & 16.155 & 16.159 \\
3.0 & 0.91079 & 0.91090 & $<0.12$ & 15.714 & 15.717 \\
4.0 & 0.79588 & 0.79609 & $<0.26$ & 14.854 & 14.860 \\
6.0 & 0.67848 & 0.67864 & $<0.24$ & 13.928 & 13.932 \\
8.0 & 0.62752 & 0.62757 & $<0.1$ & 13.675 & 13.676 \\
\hline \hline
\end{tabular}


procedures have been reviewed by Löwdin. ${ }^{33}$ In adapting these orthonormalization procedures to our problem, one readily notices that within our limited accuracy of $10^{-5}-10^{-6}$ one has to generalize the definition of lineardependent states to numerical (or practical) lineardependent states: We call a set of basis states numerically linear dependent if the measure of linear dependence (see, e.g., Courant-Hilbert ${ }^{34}$ ) is smaller than the numerical accuracy of the overlap matrix.

In the preorthonormalization procedures used by the authors, we always removed all numerically lineardependent basis states. This guarantees that all Fockmatrix elements in the orthonormalized basis are at least not totally in error. But one has to keep in mind that matrix elements involving states just above the threshold of linear dependence may have relatively large errors (well above our original accuracy of about $10^{-5}$ ). So we have to make sure that only "accurate" states contribute substantially to the total energy, otherwise we spoil its accuracy. This requirement is usually fulfilled, however, not guaranteed, especially not for optimized atomic orbitals (AO's) where significant losses of accuracy have been observed.

As this preorthonormalization is very important, we used all three principal methods described by Löwdin ${ }^{33}$ in his review article. The first method is the canonical orthonormalization where all symmetry orbitals are orthogonalized at once by diagonalization of the overlap matrix. As the canonical orthonormalization treats all symmetry orbitals on an equal footing, it is best suited if one has no additional information on the relative importance and accuracy of these basis states for the molecular orbitals.

On the other hand, we want to calculate the total energy of the system with best accuracy. As inner-shell orbitals contribute most to the total energy, and as these orbitals are less affected by the finite nuclear separation, one can argue that these symmetry states should be kept unchanged in the orthonormalization procedure.

The second method used is the Gram-Schmidt orthonormalization, where the symmetry orbitals are orthogonalized in a predefined sequence by a step-by-step procedure. We noted the symmetry orbitals according to their expectation values $\varepsilon_{\lambda}^{0}$ with some single-particle Hamiltonian $\hat{h}^{(0)}$ (e.g., the potential constructed from atomic orbitals using Mulliken occupation numbers)

$$
\varepsilon_{\lambda}^{0}=\left\langle\chi_{\lambda}\left|\hat{h}^{(0)}\right| \chi_{\lambda}\right\rangle \text {. }
$$

Starting with the lowest $\varepsilon_{1}^{0}$, the corresponding symmetry orbital $\left|\chi_{1}\right\rangle$ is taken to be the first orthogonalized symmetry orbital $\left|u_{1}\right\rangle$. Then $\left|u_{2}\right\rangle$ is constructed by orthogonalizing $\left|\chi_{2}\right\rangle$ onto $\left|\chi_{1}\right\rangle$ via a Gram-Schmidt procedure, and so on. To ensure not picking up very inaccurate states at every step, the overlap matrix $S=\left\langle\chi_{\lambda} \mid \chi_{\mu}\right\rangle \lambda, \mu=1, \ldots, k$ ( $k$ being the $k$ th step) is diagonalized. If an almost linear-dependent state $\left|\chi_{\lambda}\right\rangle$ has been picked up we will eliminate that $\left|\chi_{\lambda}\right\rangle$ and try the next $\left|\chi_{\lambda+1}\right\rangle$. In this way a reasonable set of preorthogonalized states is constructed again.

It may, however, happen that a physically important state is rejected because other, less good, basis states have already been picked up in the course of the repeated Gram-Schmidt procedure. This drawback can be remedied by a mixed selection where some $\left|\chi_{\lambda}\right\rangle$ are kept at hand. However, this may be unsatisfactory from the point of view of an automatic procedure.

The third alternative is a blockwise orthogonalization. This method was chosen mainly to allow for a mixed selection, and to ensure no loss of important physical states, as less-adapted states may cause enhanced spurious contributions. The blockwise orthogonalization causes all symmetry orbitals to split up into $M$ groups, consisting of one or more symmetry orbitals. Each group is made orthogonal on each other. If states have to be rejected they are taken from the states of the last group. This procedure guarantees that a basis can be developed, and new states can be added to a number of states used before, without any changes to the old basis.

The reason to struggle very hard for a physical basis is twofold. First, the loss of accuracy due to nonorthogonality must be kept small, because all Fock- and overlapmatrix elements are basically calculated within the AO's. There the errors in accuracy enter differently in the Fock and overlap matrix, leading to inconsistent Fock-matrix elements in the orthogonalized basis $\left|u_{\lambda}\right\rangle$, i.e., they no longer belong to the Hamiltonian $\hat{h}$, but to a modified (unphysical) one. If the amount of modification is too large, the solutions are no longer quite meaningful; therefore, we call them spurious contributions. This could be cured by computing the Fock-matrix elements in the orthogonalized states by direct integration. However, we do not yet know how to get the kinetic energy numerically with sufficient accuracy.

The second reason is-and this is just something we have learned by experience-that spurious contributions of the positron continuum are not picked up significantly in electronic states when the molecular states are sufficiently close to atomic states centered around the nuclei or the common charge center. If the orthogonalization procedure constructs completely different states, especially for the low-lying ones, this property is crucial, and we pick up spurious positron contributions in the electronic states.

Several authors ${ }^{35-43}$ have experienced large influences of the negative continuum in relativistic molecular calculations, using basis-set expansion techniques. The various attempts to avoid these problems have been reviewed by Kutzelnigg. ${ }^{15}$ It is not quite clear why the calculations presented here do not have (at least no large) spurious contributions from the negative continuum. A first attempt to explain this can be found in Ref. 44. Interesting in this connection is also the paper by Stanton and Havriliak. ${ }^{45}$ The basic theoretical papers, which deal with the general formulation of a relativistic many-particle DiracFock-Hamiltonian, are those of Mittleman, ${ }^{46,47}$ Sucher, ${ }^{48-51}$ and Grant. ${ }^{52}$

\section{RESULTS}

The DFS program under discussion here uses the local Slater approximation for the exchange term, and can, therefore, not be used generally to predict the chemical 
behavior of diatomic molecules such as accurate bond distances or binding energies, although part of the correlation is included in Slater's exchange approximation. On the other hand, however, this program has the big advantage of taking relativity fully into account. Therefore, it will be superior to accurate nonrelativistic calculations when the influence of relativity becomes strong. This is the case either at chemical distance, when very heavy atoms are involved, or already for small- $Z$ systems, when the internuclear distances are small, and inner-shell electrons influence the potential-energy curve. As an example for small internuclear distances we chose, therefore, the problem of elastic scattering of $\mathrm{Ne}$ on $\mathrm{Ne}$. As an example for chemical distances we did not choose a very heavy system but $N_{2}$. This was done because we wanted an almost fully nonrelativistic system for comparison with an analogue nonrelativistic HFS calculation. ${ }^{13}$ In addition it is interesting to see how small the relativistic influences on such a system really are.

In case of the $\mathrm{N}_{2}$ calculations ${ }^{53}$ we added the $2 p, 3 s$, and $3 p$ wave functions from $F$, the $2 s$ and $3 d$ wave functions of $\mathrm{Na}$, the $3 d$ and $4 d$ wave functions of $\mathrm{Ar}$, and the $2 s$ and $4 p$ wave functions of $\mathrm{Ti}$ to the minimum basis set around each center, and we used the Gram-Schmidt preorthogonalization. With this basis set we calculated the relativistic and nonrelativistic $(c \rightarrow \infty)$ total energy in the vicinity of the molecular bond distance as shown in Fig. 1. The minimum values at $R=2.08$ a.u. are -108.387 a.u. for the relativistic, and -108.324 a.u. for the nonrelativistic calculation. This can be compared to a fully numerical nonrelativistic HFS calculation of Laaksonen et al. ${ }^{13}$ who obtained -108.3466 a.u. at $R=2.07$ a.u. Although our basis set is not optimized for quantum-chemical calculations, our result exceeds the more exact calculations of Laaksonen ${ }^{13}$ by $0.6 \mathrm{eV}$, only.

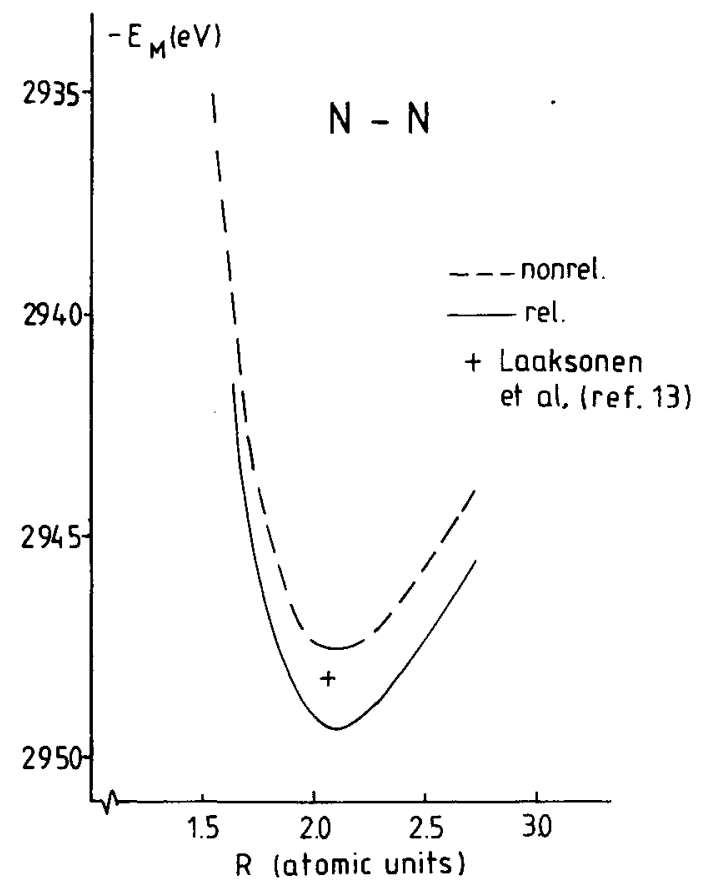

FIG. 1. Relativistic and nonrelativistic potential-energy curves for $\mathrm{N}_{2}$ as function of the internuclear distance.
TABLE III. Energy eigenvalues of the $\mathrm{N}_{2}$ molecule in a.u. at the internuclear distance $R=2.08$ a.u. The spin-orbit splitting between the $3(1 / 2)_{u}$ and $1(3 / 2)_{u}$ levels in the relativistic calculation contains a spurious contribution. It can be corrected for by the spurious contribution which appears in the nonrelativistic limit of the same calculation.

\begin{tabular}{ccccc}
\hline \hline \multicolumn{2}{c}{ State } & $\begin{array}{c}\varepsilon(\text { Nonrel. })^{\mathrm{a}} \\
\text { Ref. 13 }\end{array}$ & $\begin{array}{c}\varepsilon(\text { Nonrel. })^{\mathrm{b}} \\
\text { This work }\end{array}$ & $\begin{array}{c}\varepsilon(\text { Rel. })^{\mathrm{b}} \\
\text { This work }\end{array}$ \\
\hline $1 \sigma_{g}$ & Rel. & -13.98107 & -14.00757 & \\
$1 \sigma_{u}$ & $1(1 / 2)_{g}$ & & & -14.01627 \\
$2 \sigma_{g}$ & $1(1 / 2)_{u}$ & -13.97966 & -14.00621 & -14.01493 \\
$2 \sigma_{u}$ & $2(1 / 2)_{g}$ & -1.00721 & -1.03245 & -1.03313 \\
$1 \pi_{u}$ & $2(1 / 2)_{u}$ & -0.46072 & -0.47902 & \\
& & -0.40423 & -0.42236 & -0.47969 \\
& $3(1 / 2)_{u}$ & & -0.41753 & \\
$3 \sigma_{g}$ & $1(3 / 2)_{u}$ & & & -0.42231 \\
& $3(1 / 2)_{g}$ & -0.35006 & -0.36840 & -0.41705 \\
\hline \hline
\end{tabular}

${ }^{\mathrm{a}} R_{e}=2.07$ a.u.

${ }^{\mathrm{b}} R_{e}=2.08$ a.u.

The relativistic effect can be seen by comparing the two potential-energy curves. It results in a general decrease of $1.71 \mathrm{eV}$, and as the decrease in the total energy is $1.72 \mathrm{eV}$ in the separate atom limit, the effect on the potential energy surface is very small. Considering our numerical accuracy, we can state that relativity influences the dissociation energy by $0 \pm 0.03$, and the bond distance by $0 \pm 0.01$ a.u. The energy eigenvalues resulting from our molecular calculations are shown in Table III in comparison with the results of Laaksonen et al. ${ }^{13}$ Although our nonrelativistic values differ generally by about 0.02 a.u. from those of Ref. 13, due to our incomplete basis set the influence of relativity can be seen by comparison with our nonrelativistic calculations. The relativistic lowering of the $1 \sigma$ eigenvalue is 0.0087 a.u., which is very reasonable as this number can be compared with analogous atomic calculations.

The limits of our basis set also show up when we look at the spin-orbit splitting of the $1 \pi_{u}$-level into the relativistic $3(1 / 2) u$ and $1(3 / 2) u$ levels. Even in our nonrelativistic calculations these levels are not degenerate. Nevertheless, such a calculation is very worthwhile as it allows a good guess as to the net effect when we correct the relativistic results by subtracting the spurious nonrelativistic results. This procedure leads to a spin-orbit splitting of the $\mathrm{N}_{2} \pi$ level of $96 \mathrm{~cm}^{-1}$, which is in the correct order of magnitude.

For the system Ne-Ne we calculated ${ }^{54}$ the total energies and electron eigenvalues in the whole quasimolecular range of internuclear distances, obtaining the one-electron correlation diagram shown in Fig. 2. In these calculations 




FIG. 2. Relativistic DFS correlation diagram for Ne-Ne. The minima in the lowest MO levels are assigned by $1-3$.

we used the following basis sets: For large internuclear distances $(R>0.7$ a.u.) we added $2 s, 3 s, 3 p$ wave functions of $\mathrm{Mg}, 3 s, 3 d$ wave functions of $\mathrm{Si}, 3 d, 4 p$ wave functions of $\mathrm{Ar}$, and $3 s, 4 d$ wave functions of $\mathrm{Ni}$ to the minimum basis set; for $R \leq 0.7$ we chose the minimum basis set, and additional wave functions ( $1 s$ to $4 s$ ) from atomic calculations in the monopole potential of the two nuclei at the center of gravity of the two nuclear charges. In Fig. 3 the potential energy $V(R)$ is plotted relative to the average Lenz-Jensen potential $V_{\mathrm{aLJ}}(R)$, as suggested by Loftager et al. ${ }^{55}$ to visualize the detailed structure in the range of internuclear distances, where the atomic inner shells rearrange to form molecular orbitals. These quasimolecular potential structures can be interpreted in terms of level structures of the correlation diagram in Fig. 2. At least three internuclear distances can be found, where isolated minima in different molecular levels appear. The $2(1 / 2) \mathrm{g}$ level, e.g., has a relative minimum around $R=0.85$ a.u. with a depth of about $15 \mathrm{eV}$. The $30-\mathrm{eV}$ binding-energy contribution of this doubly occupied level leads to a relative minimum in the scaled potential at the same internuclear distance with a comparable depth. Similar arguments apply to the minimum of the $1(1 / 2) u$ and $3(1 / 2) u$ levels near $R=0.3$ a.u., and the flat minima around 0.06 a.u. in the levels which originate from the $2 p_{3 / 2}$ united atom levels. (The minima are assigned in Fig. 2.)

The quality of our quasimolecular potential calculations can be tested by comparison with experimental scattering cross sections for the system $\mathrm{Ne}^{+}-\mathrm{Ne}$, carried out by Lof-

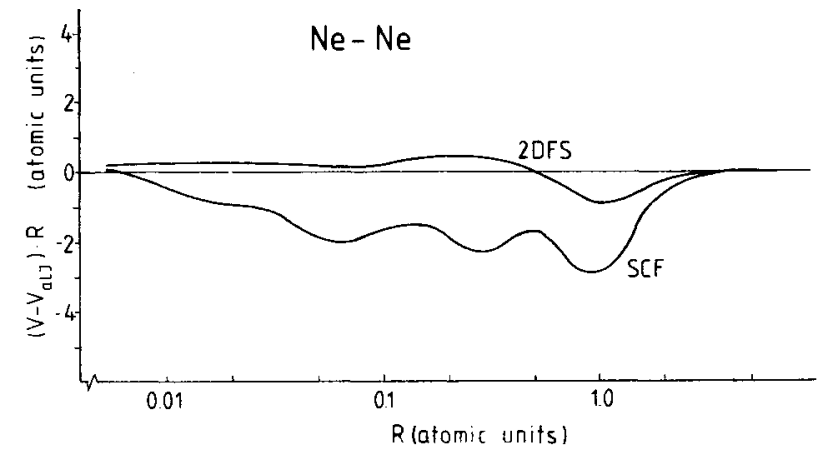

FIG. 3. Difference of the 2DFS, respectively, SCF and the averaged Lenz-Jensen (see Ref. 53) potential-energy curves. 2DFS is the statistical energy calculation using relativistic atomic densities, and SCF is the result of the relativistic quasimolecular calculation.

tager and Kristensen. ${ }^{56}$ For this reason we have calculated the differential elastic scattering cross section for our quasimolecular potential-energy surface $V(R)$ at various impact energies, and small scattering angles. The results are plotted together with the experimental values in Fig. 4. In this plot the scattering cross sections are relative to the Lenz-Jensen ${ }^{57}$ potential. The abscissa $s$ is a similarity quantity established by Lindhard, Nielsen, and Scharff (LNS theory), ${ }^{58}$ which is correlated with the distance of closest approach $R_{0}$, scaled at the top of Fig. 4 . The theoretical curve fits the experimental data quite well, and even the deviations for $R_{0}>0.6$ a.u. can be understood qualitatively. The data for $15-\mathrm{keV}$ impact energy lie systematically nearer to our adiabatic curve than the $25-\mathrm{keV}$ experimental values. The observed minimum also shifts to a larger $R_{0}$ value for smaller impact energy. So in this region the experimental cross sections are obviously influenced by inelastic effects not included in our calculation.

We discussed here only the two systems $\mathrm{N}_{2}$ and $\mathrm{Ne}-\mathrm{Ne}$ to show the quality of these calculations. Of course, it will be very worthwhile now to proceed to really heavy systems to study the influence of relativity in chemical binding. Such results will be discussed in a subsequent paper.

\section{SUMMARY AND FURTHER DEVELOPMENTS}

In this paper we presented a fully self-consistent numerical relativistic DFS code to calculate diatomic molecules at all internuclear distances. The numerical accuracy has been improved by many orders of magnitude, so that not only energy eigenvalues but also potential-energy curves emerge, allowing a quantitative interpretation. Thus the influence of relativistic effects can now be studied not only at small but also at chemical distances. This is one of the direct applications of this code probably forthcoming.

Of course, the disadvantage still is the use of the local Slater-exchange approximation. The development towards a full DF code, with exact exchange, probably is the main objective of the development in the long run, al- 


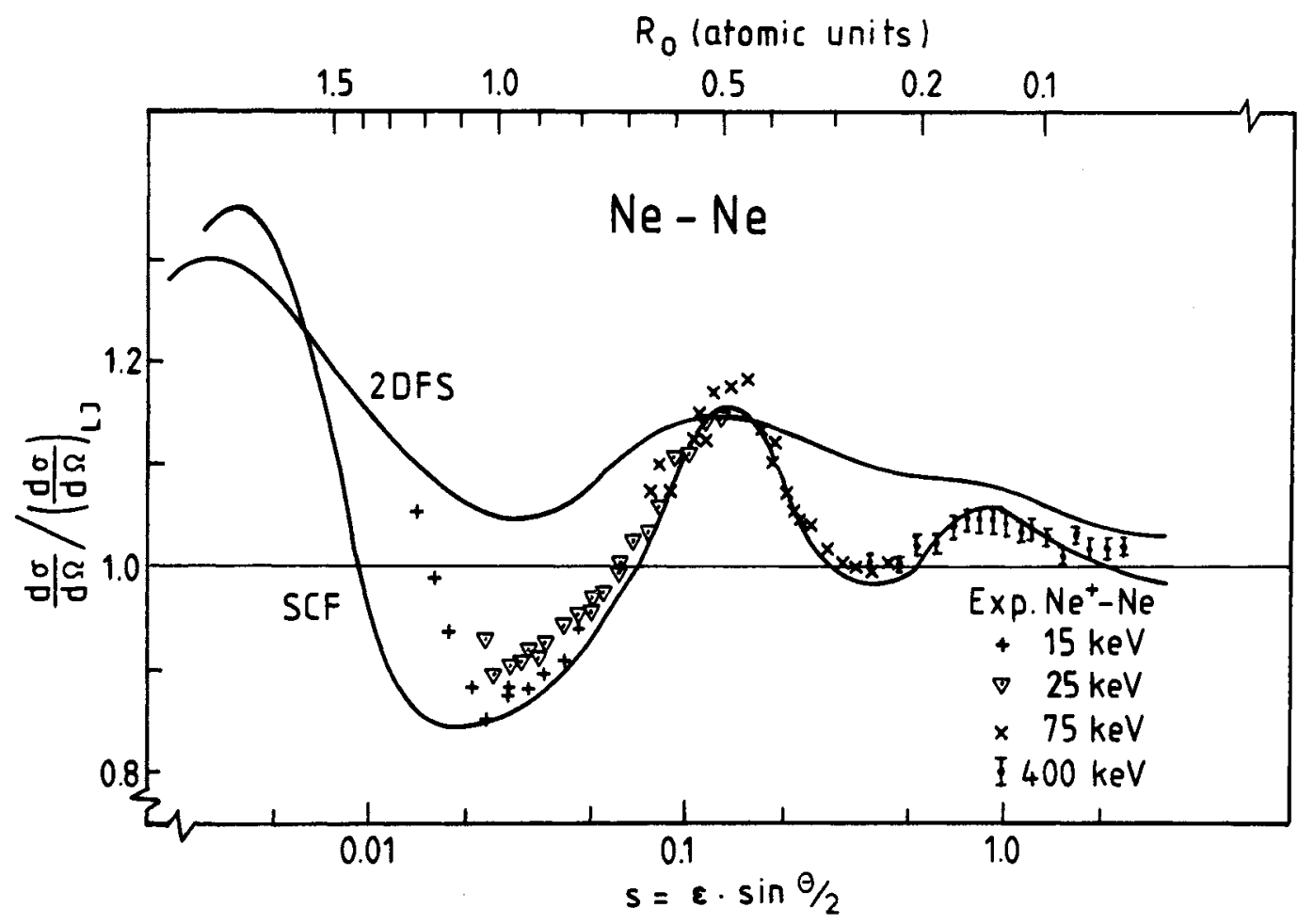

FIG. 4. Differential elastic scattering cross sections for Ne-Ne. Experimental values are taken from Refs. 54 and 57.

though it is known that results of calculations with a local exchange term are often better because part of the correlation is included. In addition, a full DF code would be too time consuming for heavy systems with the computers available at present. Therefore, in all likelihood the results of such a DFS code will be the only ones available in the region of high- $Z$ elements in the near future.

In addition to the above, several improvements have to be made to this code. One is the development of more optimized basis functions in the relativistic case. That this is of great importance has been known since the analogous nonrelativistic development many years ago. Another one is a numerical improvement to the solution of the Poisson equation. Here we hope to use the same Gauss-Laguerre grid points as for the calculations of the matrix elements. In addition, we hope to develop a new improved integration scheme where-right from the start-the already existing knowledge of the system is taken into account in the construction of the grid points, and the weights of the integration. All this might make the program a probably even more usable tool for exploring and understanding very heavy diatomic molecular systems.

\section{ACKNOWLEDGMENTS}

This work was supported in part by Gesellschaft für Schwerionenforschung (GSI), Darmstadt. The authors gratefully acknowledge Dr. A. Rosén and Dr. D. Ellis for many interesting discussions and help during this work.
${ }^{1}$ F. Herman and S. Skillman, Atomic Structure Calculations (Prentice-Hall, Englewood Cliffs, N.J., 1963).

${ }^{2}$ C. Froese-Fischer, Comput. Phys. Commun. 1, 151 (1969); 4, 107 (1972).

${ }^{3}$ A. Delgarno, Adv. Phys. 11, 281 (1962); P. L. Altik and A. E. Glasgold, Phys. Rev. 133, A632 (1964); A. Delgarno and G. A. Victor, Proc. R. Soc. London Ser. A 291, 291 (1966).

4J. P. Desclaux, Comput. Phys. Commun. 1, 216 (1969); D. A. Liberman, D. T. Cromer, and J. T. Waber, ibid. 2, 107 (1970).

${ }^{5}$ M. A. Coulthard, Proc. R. Soc. London 91, 44 (1967); J. Phys. B 6, 23 (1973); F. C. Smith and W. R. Johnson, Phys. Rev. 160, 136 (1967); Y. K. Kim, ibid. 154, 17 (1967); 159, 190 (1967); J. B. Mann, J. Chem. Phys. 51, 841 (1969); I. P. Grant, Adv. Phys. 19, 747 (1970); J. B. Mann and J. T. Waber, J. Chem. Phys. 53, 2397 (1970); J. P. Desclaux, D. F. Mayers, and F. O'Brien, J. Phys. B 4, 631 (1971); A. Rosén and I. Lindgren, Phys. Scr. 6, 24 (1972); J. Maly and M. Hussonois, Theor. Chim. Acta (Berlin) 28, 363 (1973).

6J. P. Descluax, Comput. Phys. Commun. 9, 31 (1975); I. P. Grant, B. J. McKenzie, P. H. Worrington, D. F. Mayers, and N. C. Pyper, ibid. 21, 207 (1980).

${ }^{7}$ W. R. Johnson and C. D. Lin, Phys. Rev. A 20, 964 (1979); W. R. Johnson and K. T. Cheng, ibid. 20, 978 (1979); D. Kolb, W. R. Johnson, and P. Shorer, ibid. 26, 19 (1982).

${ }^{8}$ H. F. Schaefer III, Quantum Chemistry (Clarendon, Oxford, 1984).

${ }^{9}$ L. R. Kahn and W. A. Goddard, J. Chem. Phys. 56, 2685 (1972); P. A. Christiansen, Y. S. Lee, and K. S. Pitzer, ibid. 71, 4445 (1979).

${ }^{10}$ E. A. McCullough, J. Chem. Phys. 62, 3991 (1975); P. A. 
Christiansen and E. A. McCullough, ibid. 67, 1877 (1977).

${ }^{11}$ L. Adamowicz and E. A. McCullough, J. Chem. Phys. 75;' 2475 (1981).

${ }^{12}$ A. D. Becke, J. Chem. Phys. 76, 6037 (1982).

${ }^{13}$ L. Laaksonen, D. Sundholm, and P. Pyykkö, Int. J. Quantum Chem. 27, 601 (1985).

${ }^{14}$ Relativistic Effects in Atoms, Molecules, and Solids; NATO ASI Ser. B, edited by G. L. Malli (Plenum, New York, 1983), Vol. 87.

${ }^{15}$ W. Kutzelnigg, Int. J. Quantum Chem. 25, 1076 (1984).

${ }^{16}$ W. H. E. Schwarz, S. Y. Chu, and F. Mark, Mol. Phys. 50, $603(1983)$.

${ }^{17}$ L. Laaksonen and I. P. Grant, Chem. Phys. Lett. 112, 157 (1984).

18 J. Hinze (private communication).

${ }^{19}$ J. P. Desclaux, Ref. 14, p. 213; P. Pyykkö, Adv. Quantum Chem. 11, 363 (1978); P. Pyykkö and J. P. Desclaux, Acc. Chem. Res. 12, 276 (1979).

${ }^{20}$ A. review is given by K. S. Pitzer, Ref. 14 , p. 403.

21J. G. Snijders, E. J. Baerends, and P. Ros, Mol. Phys. 38, 1909 (1979); J. G. Snijders and P. Pyykkö, Chem. Phys. Lett. 75, 5 (1980).

${ }^{22} \mathrm{~A}$ review is given by C. Y. Yang, Ref. 14, p. 335.

${ }^{23}$ A. Rosén and D. E. Ellis, J. Chem. Phys. 62, 3039 (1975).

${ }^{24}$ F. W. Averill and D. E. Ellis, J. Chem. Phys. 59, 6412 (1973).

${ }^{25}$ D. E. Ellis and G. S. Painter, Phys. Rev. B 2, 2887 (1970).

${ }^{26}$ D. E. Ellis and A. Rosén, Z. Phys. A 283, 3 (1977); J. Paul and A. Rosén, Phys. Rev. B 26, 4073 (1982).

${ }^{27}$ B. Fricke, W.-D. Sepp, and T. Morović, Phys. Lett. 81A, 258 (1981); J. Phys. B 14, L549 (1981).

${ }^{28}$ B. Delley and D. E. Ellis, J. Chem. Phys. 76, 1949 (1982).

${ }^{29}$ A. Rosén, D. E. Ellis, H. Adachi, and F. W. Averill, J. Chem. Phys. 65, 3629 (1976).

${ }^{30}$ G. E. Brown and D. G. Ravenhall, Proc. R. Soc. London Ser. A 208, 552 (1951).

${ }^{31}$ W. Betz, Ph.D. dissertation, University of Frankfurt am Main, 1980.

${ }^{32}$ P. N. Scharztrauber and R. A. Sweet, ACM Trans. Math. Software 5, 352 (1979).

${ }^{33}$ P. O. Löwdin, Adv. Phys. 5, 1 (1956).

${ }^{34} \mathrm{R}$. Courant and D. Hilbert, Methoden der Mathematischen Physik (Springer-Verlag, Berlin, 1931), p. 51.

${ }^{35}$ F. Mark, H. Lischka, and F. Rosicky, Chem. Phys. Lett. 71, 507 (1980).

${ }^{36}$ F. Mark and F. Rosicky, Chem. Phys. Lett. 74, 562 (1980).
${ }^{37}$ H. Wallmeier and W. Kutzelnigg, Chem. Phys. Lett. 78, 341 (1981).

${ }^{38}$ G. Malli and J. Oreg, Chem. Phys. Lett. 69, 313 (1979).

${ }^{39}$ O. Matsuoka, N. Suzuki, T. Aoyana, and G. Malli, J. Chem. Phys. 73, 1320 (1980).

${ }^{40}$ T. Aoyana, H. Yamakawa, and O. Matsuoka, J. Chem. Phys. 73, 1329 (1980).

${ }^{41}$ S. N. Datta and C. S. Ewig, Chem. Phys. Lett. 85, 443 (1982).

${ }^{42}$ Y. S. Lee and A. D. McLean, J. Chem. Phys. 76, 735 (1982).

${ }^{43}$ W. H. E. Schwarz and E. Wechsel-Trakowski, Chem. Phys. Lett. 85, 94 (1982).

${ }^{44}$ W.-D. Sepp and B. Fricke, Proceedings of the Atomic Theory Workshop on Relativistic and QED Effects in Heavy Atoms, Gaithersburg, 1985, edited by H. P. Kelly and Y.-K. Kim (AIP, New York, 1985), p. 20.

${ }^{45}$ R. E. Stanton and S. Havriliak, J. Chem. Phys. 81, 1910 (1984).

${ }^{46}$ M. H. Mittleman, Phys. Rev. A 4, 893 (1971); 15, 2395 (1972).

${ }^{47}$ M. H. Mittleman, Proceedings of the Workshop on Foundations of the Relativistic Theory of Atomic Structures, edited by H. G. Berry, K. T. Cheng, W. R. Johnson, and Y.-K. Kim [Argonne National Laboratory Report ANL-80-126, 1981 (unpublished), p. 27.]

${ }^{48}$ G. Feinberg and J. Sucher, Phys. Rev. Lett. 26, 681 (1971).

49J. Sucher, Rep. Prog. Phys. 41, 1781 (1978).

${ }^{50} \mathrm{~J}$. Sucher, Proceedings of the Workshop on Foundations of the Relativistic Theory of Atomic Structures, edited by H. G. Berry, K. T. Cheng, W. R. Johnson, and Y.-K. Kim [Argonne National Laboratory Report ANL-80-126, 1981 (unpublished), p. 1.]

51 J. Sucher, in Ref. 14, p. 1.

${ }^{52}$ I. P. Grant, in Ref. 14, p. 73.

${ }^{53}$ W.-D. Sepp, W. Sengler, D. Kolb, H. Hartung, and B. Fricke, Chem. Phys. Lett. 109, 233 (1984).

${ }^{54}$ H. Hartung, B. Fricke, W.-D. Sepp, W. Sengler, and D. Kolb, J. Phys. B 18, L433 (1979).

${ }^{55}$ P. Loftager, F. Besenbacher, O. S. Jensen, and V. S. Sørensen; Phys. Rev. A 20, 1443 (1979).

${ }^{56}$ P. Loftager and P. Kristensen, Institute of Physics 1979/80 Annual Report (University of Aarhus, Aarhus, Denmark, 1981), p. 83.

${ }^{57}$ H. Lenz, Z. Phys. 77, 713 (1932); H. Jensen, ibid. 77, 722 (1932).

${ }^{58}$ J. Lindhard, V. Nielsen, and M. Scharff; K. Dan. Vidensk. Selsk. Mat.-Fys. Medd. 36, no. 10 (1968). 